\title{
Comparison of medial femoral cartilage deformation in normal adults according to gait conditions
}

\author{
Sang-Cheol Im, Kyoung Kim* \\ Department of Physical Therapy, College of Rehabilitation Science, Daegu University, Gyeongsan, Korea
}

This study aimed to explore the deformation of medial femoral cartilage in normal adults according to gait conditions. Overall, 76 normal adults without degenerative arthritis or a knee injury on medical history were randomly assigned into control, flatland walk, slope walk, and stepper walk groups. The control group was rested for $30 \mathrm{~min}$, the test group performed flatland walking, $16^{\circ}$ slope walking, and stepper walking, respectively. The thickness of medial femoral cartilage before and after gait was evaluated through ultrasound test. Compared with the control group, a significant difference was noted for medial femoral cartilage deformation before and after gait in all the three groups. Comparison of the medial femoral cartilage deformation among the groups revealed a significant difference between the control group and the flatland walk, slope walk, and stepper walk groups $(P<0.05)$. The flatland walk group had a significant difference between the slope walk group and stepper walk group $(P<0.05)$, whereas no significant difference was noted between the slope walk and stepper walk groups $(P>0.05)$. After a 30-min walk, the thickness of medial femoral cartilage was reduced, and a difference in deformation was noted according to gait conditions. The thickness of medial femoral cartilage was reduced more in the stepper walk and slope walk groups, wherein more load operates on the knee, than the flatland walk group.

Keywords: Medial femoral cartilage, Ultrasound, Gait, Cartilage thickness, Knee

\section{INTRODUCTION}

Recent developments in medical technology have resulted in an increase in life expectancy such that the ratio of old population has been increasing. Therefore, the number of patients with degenerative knee arthritis has also been increasing. Degenerative knee arthritis is characterized by a structural change in the knee joint, including the joint cartilage and neighboring bones, and by synovial inflammation and meniscus damage (Loeser et al., 2012). It is the sixth leading cause of disability worldwide (Glyn-Jones et al., 2015). The earliest stage of degenerative arthritis appears with a change in formation tissue before the reduction of cartilage structure (Guermazi et al., 2015) and gradually leads to the reduction of cartilage thickness (Eckstein et al., 2015). Reduction in joint space, which is visualized by $\mathrm{X}$-ray, is considered as the reduction of cartilage thickness and is used for the diagnosis of degenerative knee arthritis (Hunter et al., 2006).
However, X-ray has a limitation because it cannot visualize joint cartilage directly and evaluates cartilage indirectly through the joint space (gap) (Oei et al., 2018). Therefore, a more sensitive image diagnosis method is necessary to visualize the joint cartilage directly and observe a change in detail (Harkey et al., 2017). Magnetic resonance imaging (MRI) can evaluate knee joint cartilage with accuracy and reliability, but it is expensive and time consuming. Accordingly, recently, ultrasound test, which is considered as a valid and reliable technology and can evaluate the thickness of medial femoral cartilage, is gaining attention (Naredo et al., 2009). Ultrasound test is a useful technique for diagnosing not only joint cartilage, muscle, ligament, meniscus, and lesions in the synovial space and muscle but also knee joint diseases. It is noninvasive, extensively available, can be performed swiftly, and can be used easily without patient reluctance. Moreover, there is no radiation exposure, and it is cheaper than MRI (Lee and Chow, 2007). A comparative study between ultrasound and MRI, ultra-
${ }^{*}$ Corresponding author: Kyoung Kim (iD https://orcid.org/0000-0003-4169-6852 Department of Physical Therapy, College of Rehabilitation Science, Daegu University, 201 Daegudae-ro, Jillyang-eup, Gyeongsan 38453, Korea E-mail: kykim257@hanmail.net

Received: April 10, 2019 / Accepted: June 4, 2019
This is an Open Access article distributed under the terms of the Creative Commons Attribution Non-Commercial License (http://creativecommons.org/licenses/by-nc/4.0/) which permits unrestricted non-commercial use, distribution, and reproduction in any medium, provided the original work is properly cited. 
sound demonstrated high reliability and was considered a useful tool for evaluating the thickness of medial femoral cartilage quantitatively (Ostergaard et al., 1995).

Various biomechanical loads that operate on knee joint can interrupt the homeostasis of normal cartilage and markedly influence the outbreak and progress of degenerative knee arthritis (Lad et al., 2016; Sutter et al., 2015). The measurement of joint cartilage thickness can give an estimate of the general cartilage structure (Harkey et al., 2017) to provide more important information than that provided by measuring the cartilage thickness that has a stable condition to evaluate the change in joint cartilage thickness immediately following everyday life activity. Therefore, if ultrasound test is used for quantifying how normal cartilage responds to everyday life activity, a clinical tool that can evaluated the early changes in cartilage condition can be developed (Sutter et al., 2015).

Several previous studies that have evaluated the instant change in knee joint cartilage thickness according to various loads of normal joint have been reported. Lad et al. (2016) targeting normal individuals and reported the reduction of cartilage thickness in the MRI assessment of knee joint before and after a 20-min walk. With ultrasound, Harkey et al. (2017) evaluated cartilage thickness immediately after a 30-min walk and run in healthy individuals. There was a significant reduction in cartilage thickness in the walk and running group than in control group who rested for $30 \mathrm{~min}$, and there was no significant difference between the two groups. Boocock et al. (2009) reported that the volume and thickness of knee joint cartilage reduced after varying time intervals and distances of running exercise. Moreover, cartilage deformation and its extent or amount varies according to activities, including running and landing from a certain height (Niehoff et al., 2011).

As mentioned above, normal knee joint cartilage responds to biomechanical loads that are applied during everyday life activity. In addition, walking is the most universal activity in everyday life. Therefore, it is important to examine the instant change in medial femoral cartilage after walking in normal adults to evaluate the response of impaired cartilage in diseased patients. However, research and studies on cartilage deformation caused by various biomechanical loads on knee joint are limited. In particular, slope and stair climbing are the second most frequent walking environments in everyday life after flatland walking. Therefore, the present study aimed to compare the deformation of medial femoral cartilage in normal adults using ultrasound and based on various gait conditions that have not been explored much in the literature.

\section{MATERIALS AND METHODS}

\section{Participants}

This research targeted normal adults aged 20-35 years who reported that they participate in physical activity for at least for 20 $\min$ for 3 days a week. Selection criteria were individuals without knee joint arthritis, leg surgery, knee ligament injury, chronic ankle joint instability, and medical history or symptom of balance disability for 6 months before the experiment, they could understand and follow the directions of a researcher, and provides consent for participation in the study. It was considered necessary to include at least 76 individuals to evaluated a significance level of $0.05,80 \%$ of power, using G-power (V3.1) (Harkey et al., 2018). Using the computer program, a total of 76 people were randomly assigned to control $(\mathrm{n}=19)$, flatland walk $(\mathrm{n}=19)$, slope walk $(\mathrm{n}=$ $19)$, and stepper $(\mathrm{n}=19)$ groups. This research was approved by the Bioethics Committee of Daegu University (1040621-201811HR-019-02) and received written consent of all participants before the experiment.

\section{Assessment tool and measurement method Ultrasound test}

The thickness of femoral joint cartilage was evaluated by an examiner by evaluating the ultrasonic image of every femoral joint cartilage using the LS128 US (Telemed, Savanoriu, Vilnius, Lithuania) system, which has a 12-MHz linear probe. The thickness of femoral joint cartilage was measured in the dominant legs as reported by the participants. They placed their backs on a wall and took a posture in which their knee joints were bent at $140^{\circ}$ using a manual joint goniometer (Fig. 1). To guarantee similar posture among the participants in the posttest, the distance between the wall and heel was measured and recorded, and a tape was placed

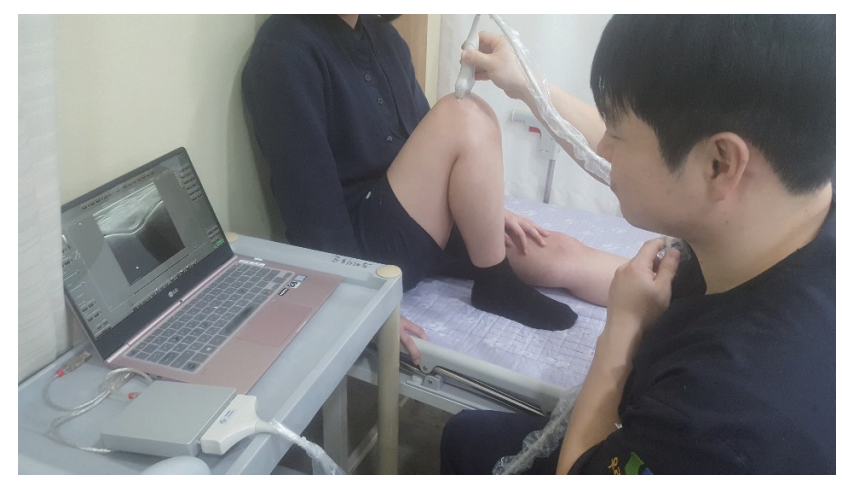

Fig. 1. Subject setup and ultrasound probe positioning. Informed consent for publication was obtained from subjects. 


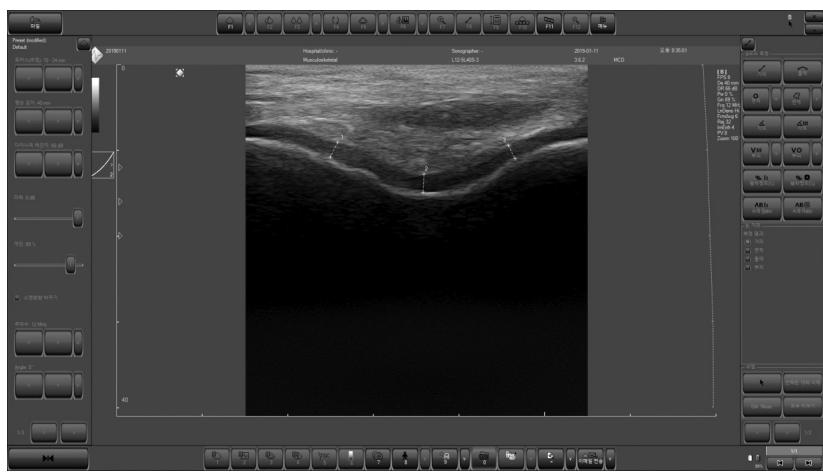

Fig. 2. Ultrasonographic image of the femoral articular cartilage.

and marked at the test table. A probe was intersected straight from the upper edge of patella bone to the medial and lateral condyle of femur and was rotated to maximize the reflection of joint cartilage surface (Naredo et al., 2009; Özçakar et al., 2014; Tuna et al., 2016). To enhance the reproducibility of the ultrasound test, a transparent acryl grid was arranged on the display screen. If an intercondylar notch is placed at the center of the acryl grid, the location of the medial and lateral condyle of femur in the edge of the screen is marked. The location is repeated in posttest, and it guarantees a similar probe arrangement in consecutive ultrasound tests. Three images were recorded for each participant.

\section{Analysis of ultrasound images}

Ultrasound images were analyzed with ECO WAVE II (Telemed) software by an examiner. The thickness of femoral joint cartilage was measured as the straight distance between the cartilage-bone interface and the synovia-cartilage interface and estimated at the midpoint of the medial condyle of the femur (Naredo et al., 2009; Özçakar et al., 2014). The average for statistical analysis was calculated in the three images of each participant, which were obtained before and after gait (Fig. 2).

\section{Materials and methods}

\section{Experiment plan}

This study measured the thickness of medial femoral cartilage in normal adults who walked for 30 min before walk under stable conditions and three gait conditions. The participants were directed to limit their physical activities on the day of data collection. Ultrasound test was performed for dominant legs, which the participants used to kick a ball. All the experiments were performed under the supervision of the researcher. Ultrasound test was performed by an expert examiner. The examiner was unaware of the
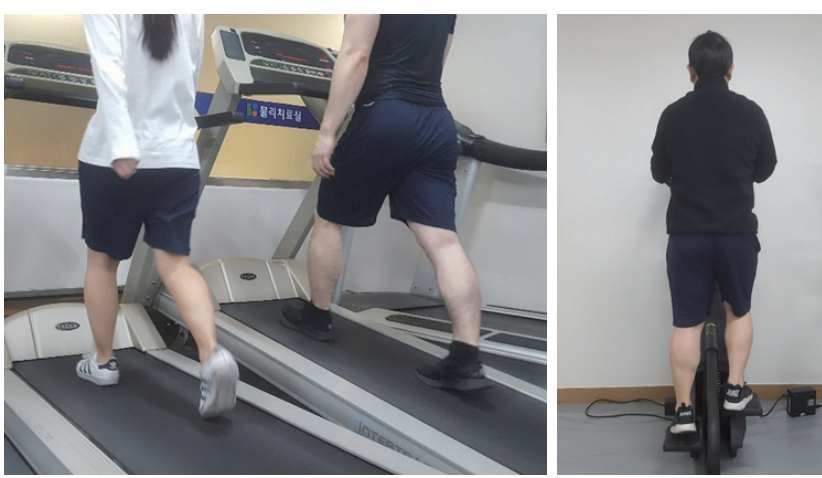

Fig. 3. Gait conditions. Informed consent for publication was obtained from subjects.

gait conditions that the participants had followed before the ultrasound test.

\section{Experiment method}

On their arrival in the experiment room, all the participants spread their knees on the text table completely on a flat surface, put their backs against the wall, and rested for 30 min to remove the load of femoral joint cartilage and minimize the influence of the preceding activities (Niehoff et al., 2011). If the rest was over, ultrasound test was performed to measure the thickness of femoral joint cartilage under stable conditions. After the preultrasound test, participants began to walk for $30 \mathrm{~min}$ immediately on the treadmill and stepper, which were in the same experiment room (Fig. 3). The control group $(n=19)$ rested at the table for $30 \mathrm{~min}$, the flatland walk group $(\mathrm{n}=19)$ walked on the treadmill without a slope, the slope walk group $(n=19)$ walked on the treadmill with a $16^{\circ}$ slope, and the stepper walk group $(n=19)$ walked on a stepper wherein crossover exercises of both legs could be performed similar to stair climbing (Choi et al., 2015). Gait speed on the treadmill and stepper was self-chosen speed in which maximum descent ranging from 3 to 4 points was maintained with Rating of Perceived Exertion (Borg scale). After the 30-min walk, participants moved to the test table instantly for postultrasound assessment. Using the tape attached to the test table, participants took the same posture as that during the preultrasound test, and three ultrasound images of femoral joint cartilage were recorded through the same procedure. All postultrasound assessments were performed within 5 min before they stopped walking.

\section{Analysis method}

The data collected in this study were statistically processed with window statistical processing program, IBM SPSS ver. 18.0 
(IBM Co., Armonk, NY, USA). The basic characteristics of the participants were analyzed by technical statistics and Kolmogorov-Smirnov test was used for the regularity verification of all data. One-way analysis of variance was used to evaluate the difference in the basic characteristics of each group and to evaluate the difference in the change of medial femoral cartilage among gait conditions. Paired samples $t$-test was used to evaluate the change in medial femoral cartilage before and after gait. Least square difference (LSD) was used for post examination to explain the difference in gait conditions. $P<0.05$ was considered statistically significant.

\section{RESULTS}

\section{Basic characteristics of the participants}

A total of 76 participants were included in this study. These participants were assigned to four groups ( $\mathrm{n}=19$ in each group). The basic characteristics are summarized in Table 1. No significant differences were noted in these characteristics among each group $(P>0.05$; Table 1$)$.

\section{Comparison of medial femoral cartilage deformation according to gait conditions}

There was no significant difference among the four groups in terms of thickness of medial femoral cartilage before gait $(P>0.05$; Table 1). However, a significant difference was noted among all the four groups in terms of medial femoral cartilage deformation before and after gait between the control group and the three gait condition groups $(P<0.05$; Table 2$)$. The comparison of medial femoral cartilage deformation among the groups according to gait conditions revealed a significant difference $(P<0.05$; Table 2$)$.

The result of LSD post examination, which was conducted for explaining the deformation difference of medial femoral cartilage according to gait conditions, was that there was a significant difference between control group and flatland walk group, slope walk group and stepper walk group $(P<0.05$; Table 3$)$. There was a significant difference between flatland walk group and slope walk group and stepper walk group $(P<0.05$; Table 3$)$ and there was not a significant difference between slope walk group and stepper walk group $(P>0.05$; Table 3$)$.

\section{DISCUSSION}

Joint cartilage plays a role in decreasing frictional force during joint exercises, and biomechanical factors play an important role in maintaining the health of joints (Andriacchi et al., 2009). Cartilage load can also result in the impairement and loss of joint

Table 1. General characteristics of subjects

\begin{tabular}{|c|c|c|c|c|c|}
\hline Characteristic & $C G(n=19)$ & EG1 $(n=19)$ & $\mathrm{EG} 2(\mathrm{n}=19)$ & $\mathrm{EG} 3(n=19)$ & $P$-value \\
\hline Gender, male:female & 8:11 & 6:13 & $7: 12$ & 9:10 & \\
\hline Age (yr) & $26.59 \pm 3.65$ & $27.78 \pm 3.44$ & $27.21 \pm 3.13$ & $26.47 \pm 3.83$ & 0.60 \\
\hline Height (cm) & $167.78 \pm 8.05$ & $166.52 \pm 8.64$ & $168.42 \pm 7.49$ & $168.89 \pm 6.96$ & 0.78 \\
\hline Weight (kg) & $66.94 \pm 10.98$ & $64.31 \pm 14.15$ & $62.47 \pm 12.43$ & $67.05 \pm 14.01$ & 0.64 \\
\hline Body mass index $\left(\mathrm{kg} / \mathrm{m}^{2}\right)$ & $27.09 \pm 7.74$ & $25.53 \pm 10.51$ & $23.72 \pm 8.88$ & $27.42 \pm 11.04$ & 0.59 \\
\hline
\end{tabular}

Values are presented as mean \pm standard deviation.

CG, rest group; $E G 1$, level walking group; $E G 2,16^{\circ}$ ascending walking group; $E G 3$, stepper walking group.

Table 2. Comparison of the medial femoral cartilage thickness of the four groups

\begin{tabular}{|c|c|c|c|c|c|}
\hline MFC & $C G(n=19)$ & $\mathrm{EG1}(\mathrm{n}=19)$ & $\mathrm{EG} 2(\mathrm{n}=19)$ & $\mathrm{EG} 3(\mathrm{n}=19)$ & $P$-value \\
\hline Walking speed (m/sec) & - & $1.46 \pm 0.17$ & $0.92 \pm 0.14$ & $0.62 \pm 0.08$ & - \\
\hline Walking distance (km) & - & $2.64 \pm 0.31$ & $1.66 \pm 0.26$ & $1.13 \pm 0.15$ & - \\
\hline Pre (mm) & $1.96 \pm 0.29$ & $2.13 \pm 0.31$ & $2.04 \pm 0.31$ & $1.98 \pm 0.29$ & 0.31 \\
\hline Post (mm) & $2.08 \pm 0.28$ & $2.00 \pm 0.29$ & $1.88 \pm 0.28$ & $1.79 \pm 0.25$ & - \\
\hline Absolute change (mm) & $0.12 \pm 0.04$ & $-0.12 \pm 0.02$ & $-0.16 \pm 0.03$ & $-0.19 \pm 0.04$ & $0.00^{*}$ \\
\hline Percent change (\%) & $6.31 \pm 2.62$ & $-5.87 \pm 0.77$ & $-8.22 \pm 0.97$ & $-9.45 \pm 1.60$ & - \\
\hline$t$ & 12.84 & -18.47 & -21.78 & -15.62 & - \\
\hline$P$-value & $0.00^{*}$ & $0.00^{*}$ & $0.00^{*}$ & $0.00 *$ & - \\
\hline
\end{tabular}

Values are presented as mean \pm standard deviation.

MFC, medial femoral cartilage; CG, rest group; EG1, level walking group; EG2, $16^{\circ}$ ascending walking group; EG3, stepper walking group.

${ }^{*} P<0.05$. 
Table 3. Post hoc tests of the medial femoral cartilage thickness in each group

\begin{tabular}{llccc}
\hline Group & & MD & SE & P-value \\
\hline CG & EG1 & 0.247 & 0.013 & $0.00^{*}$ \\
& EG2 & 0.290 & 0.013 & $0.00^{*}$ \\
& EG3 & 0.309 & 0.013 & $0.00^{*}$ \\
EG1 & CG & -0.247 & 0.013 & $0.00^{*}$ \\
& EG2 & 0.042 & 0.013 & $0.00^{*}$ \\
& EG3 & 0.061 & 0.013 & $0.00^{*}$ \\
& CG & -0.290 & 0.013 & $0.00^{*}$ \\
& EG1 & -0.042 & 0.013 & $0.00^{*}$ \\
EG3 & EG3 & 0.019 & 0.013 & 0.14 \\
& CG & -0.309 & 0.013 & $0.00^{*}$ \\
& EG1 & -0.061 & 0.013 & $0.00^{*}$ \\
& EG2 & -0.019 & 0.013 & 0.14 \\
\hline
\end{tabular}

$\mathrm{MD}$, mean difference; $\mathrm{SD}$, standard error; $\mathrm{CG}$, rest group; $\mathrm{EG1}$, level walking group; $\mathrm{EG} 2,16^{\circ}$ ascending walking group; EG3, stepper walking group.

${ }^{*} P<0.05$.

function, which is a characterisitc of degenerative arthritis (Arokoski et al., 2000). Abnormal load can be an important factor of degenerative arthritis progress (Kumar et al., 2013); however, its accurate mechanism remains unknown. The response of normal cartilage must be elucidated to understand the response of impaired cartilage to the load that operates on the joint cartilage in diseased patients. Therefore, this research targeted normal adults; conducted an experiment in which necessary activities of everyday life, including walking, were performed under various conditions; and aimed to evaluate medial femoral cartilage deformation according to gait conditions.

The results of this study revealed that, in posttest, a significant difference was noted in medial femoral cartilage deformation between the control group and the three gait condition groups. The thickness of medial femoral cartilage in the control group, which was rested for $30 \mathrm{~min}$, increased by $6.31 \%$. A previous study reported that if pressure is removed in joint cartilage, a fluid is reabsorbed over time due to the low permeability of cartilage tissue; this finding is consistent with that reported in our study (Herberhold et al., 1999). In addition, it showed a bigger change than that reported in the study by Niehoff et al. (2011), wchih used MRI accessments and reported that the thickness of medial femoral cartilage in the group rested for $30 \mathrm{~min}$ increased by $2.6 \%$. This difference is possibly caused because of the difference in posture during rest. In the study of Niehoff et al. (2011), participants rested while they sat on chairs and placed their legs on the floor. However, in our study, participants rested while they stretched out their legs on tables, indicating that the rest posture in this study reduced more load on the knee joint.
In the flatland walk group, the thickness of medial femoral cartilage decreased by $5.87 \%$ in posttest. Lad et al. (2016) reported that medial femoral cartilage was reduced by $1.2 \%$ after a $20-\mathrm{min}$ walk on the treadmill, whereas in the present study, it reduced by $5.87 \%$ in the flatland walk group, indicating a larger deformation than that reported by Lad et al. (2016). This difference could possibly be due to the difference in walking time and assessment tools. In this study, the walking time was 10 min longer; therefore, more load was operated on the knee joint, affecting the joint cartilage. Moreover, it is believed that ultrasound provided postassessment results faster than that by provided by MRI. In this study, all the post-assessments could be performed within $5 \mathrm{~min}$ after the 30-min walk because the test table, which was used for ultrasound assessment, was located right next to the treadmill and stepper, which were used for walking. Therefore, in comparison with the study that used MRI, the present study could confirm cartilage deformation early at recovery stage after the end of the 30-min walk; indicating that it showed bigger deformation than the study that used MRI. Medial femoral cartilage in the slope and stepper walk groups was reduced by $8.22 \%$ and $9.45 \%$, respectively, suggesting more reduction than that in the flatland walk group.

In the comparison among groups, significant differences were noted under gait conditions. As a result of postanalysis, the thickness of medial femoral cartilage increased in the control group who rested, whereas it decreased in the three groups under gait conditions; this result was significant. Regarding comparisons among the gait conditions, in the flatland walk group, a significant difference between the slope and stepper walk groups in flatland walk group and more reduction was shown in slope walk group and stepper walk group. There was no significant difference between the slope and stepper walk groups. The uphill slope walking makes outbreak of cartilage impairment higher than that with flatland walking because the operating range of knee joint angle is decreased and the strength of knee joint is increased. Bennett et al. (2018) reported that stair climbing imposes bigger loads and moments. Accordingly, in slope and stepper walking in which bigger loads operate on knee joint than flatland walking, it is considered that the thickness of medial femoral cartilage is reduced more.

This research has several limitations. First, the sample size is small for generalization. Therefore, further research with a larger sample size focusing on the response of abnormal joint cartilage of patients knee joint diseases in normal adults. Second, ultrasound test is a promptly approachable method, which can notice the de- 
formation of medial femoral cartilage early. However, it could only evaluate the front part of medial femoral cartilage in comparison with the MRI test, which can observe average thickness change of joint cartilage and could not measure the thickness of joint cartilage between shinbone and femur and between knee bone and femur. Third, this research evaluated only the thickness of medial femoral cartilage, and it is necessary to have an additional study on the different divisions of femoral joint cartilage. Fourth, in this research, people walked with self-chosen speed. However, it is necessary to have a research which quantifies speed and distance in the future. Fifth, stepper walk is similar to stairs climbing, but could be different from real stairs climbing in terms of the response of joint cartilage. So, it is necessary to study the response of joint cartilage after real stair climbing.

In conclusion, ultrasound test is a tool which can evaluate promptly the medial femoral cartilage of normal adults and is sensitive to the instant deformation of joint cartilage after walk. After 30-minwalk, thickness of medial femoral cartilage reduced and there was a difference according to gait conditions. In stepper walk and slope walk in which bigger loads operate on knee joint, thickness of joint cartilage reduced more than flatland walk.

\section{CONFLICT OF INTEREST}

No potential conflict of interest relevant to this article was reported.

\section{REFERENCES}

Andriacchi TP, Koo S, Scanlan SF. Gait mechanics influence healthy cartilage morphology and osteoarthritis of the knee. J Bone Joint Surg Am 2009;9 Suppl 1:95-101.

Arokoski JP, Jurvelin JS, Väätäinen U, Helminen HJ. Normal and pathological adaptations of articular cartilage to joint loading. Scand J Med Sci Sports 2000;10:186-198.

Bennett HJ, Weinhandl JT, Fleenor K, Zhang S. Frontal plane tibiofemoral alignment is strongly related to compartmental knee joint contact forces and muscle control strategies during stair ascent. J Biomech Eng 2018.

Boocock M, McNair P, Cicuttini F, Stuart A, Sinclair T. The short-term effects of running on the deformation of knee articular cartilage and its relationship to biomechanical loads at the knee. Osteoarthritis Cartilage 2009;17:883-890.

Choi M, Yoo J, Shin S, Lee W. The effects of stepper exercise with visual feedback on strength, walking, and stair climbing in individuals fol- lowing stroke. J Phys Ther Sci 2015;27:1861-1864.

Eckstein F, Collins JE, Nevitt MC, Lynch JA, Kraus VB, Katz JN, Losina E, Wirth W, Guermazi A, Roemer FW, Hunter DJ; FNIH OA Biomarkers Consortium. Brief report: cartilage thickness change as an imaging biomarker of knee osteoarthritis progression: data from the Foundation for the National Institutes of Health Osteoarthritis Biomarkers Consortium. Arthritis Rheumatol 2015;67:3184-3189.

Glyn-Jones S, Palmer AJ, Agricola R, Price AJ, Vincent TL, Weinans H, Carr AJ. Osteoarthritis. Lancet 2015;386:376-387.

Guermazi A, Alizai H, Crema MD, Trattnig S, Regatte RR, Roemer FW. Compositional MRI techniques for evaluation of cartilage degeneration in osteoarthritis. Osteoarthritis Cartilage 2015;23:1639-1653.

Harkey MS, Blackburn JT, Davis H, Sierra-Arévalo L, Nissman D, Pietrosimone B. Ultrasonographic assessment of medial femoral cartilage deformation acutely following walking and running. Osteoarthritis Cartilage 2017;25:907-913.

Harkey MS, Blackburn JT, Davis H, Sierra-Arévalo L, Nissman D, Pietrosimone $B$. The association between habitual walking speed and medial femoral cartilage deformation following 30minutes of walking. Gait Posture 2018:128-133.

Herberhold C, Faber S, Stammberger T, Steinlechner M, Putz R, Englmeier KH, Reiser M, Eckstein F. In situ measurement of articular cartilage deformation in intact femoropatellar joints under static loading. J Biomech 1999;32:1287-1295.

Hunter DJ, Zhang YQ, Tu X, Lavalley M, Niu JB, Amin S, Guermazi A, Genant $\mathrm{H}$, Gale D, Felson DT. Change in joint space width: hyaline articular cartilage loss or alteration in meniscus? Arthritis Rheum 2006; 54:2488-2495.

Kumar D, Manal KT, Rudolph KS. Knee joint loading during gait in healthy controls and individuals with knee osteoarthritis. Osteoarthritis Cartilage 2013;21:298-305.

Lad NK, Liu B, Ganapathy PK, Utturkar GM, Sutter EG, Moorman CT 3rd, Garrett WE, Spritzer CE, DeFrate LE. Effect of normal gait on in vivo tibiofemoral cartilage strains. J Biomech 2016;49:2870-2876.

Lee MJ, Chow K. Ultrasound of the knee. Semin Musculoskelet Radiol 2007;11:137-148.

Loeser RF, Goldring SR, Scanzello CR, Goldring MB. Osteoarthritis: a disease of the joint as an organ. Arthritis Rheum 2012;64:1697-1707.

Naredo E, Acebes C, Möller I, Canillas F, de Agustín JJ, de Miguel E, Filippucci E, Iagnocco A, Moragues C, Tuneu R, Uson J, Garrido J, Delgado-Baeza E, Sáenz-Navarro I. Ultrasound validity in the measurement of knee cartilage thickness. Ann Rheum Dis 2009;68:1322-1327.

Niehoff A, Müller M, Brüggemann L, Savage T, Zaucke F, Eckstein F, Müller-Lung U, Brüggemann GP. Deformational behaviour of knee cartilage and changes in serum cartilage oligomeric matrix protein 
(COMP) after running and drop landing. Osteoarthritis Cartilage 2011;19:1003-1010.

Oei EHG, Wick MC, Müller-Lutz A, Schleich C, Miese FR. Cartilage imaging: Techniques and developments. Semin Musculoskelet Radiol 2018;22:245-260.

Ostergaard M, Court-Payen M, Gideon P, Wieslander S, Cortsen M, Lorenzen I, Henriksen O. Ultrasonography in arthritis of the knee. A comparison with MR imaging. Acta Radiol 1995;36:19-26.

Özçakar L, Tunç H, Öken Ö, Ünlü Z, Durmuş B, Baysal Ö, Altay Z, Tok F, Akkaya N, Doğu B, Çapkın E, Bardak A, Çarlı AB, Buğdaycı D, Toktaş H, Diraçoğlu D, Gündüz B, Erhan B, Kocabaş H, Erden G, Günendi Z, Kesikburun S, Omaç ÖK, Taşkaynatan MA, Şenel K, Uğur M, Yalçınkaya
EY, Öneş K, Atan Ç, Akgün K, Bilgici A, Kuru Ö, Özgöçmen S. Femoral cartilage thickness measurements in healthy individuals: learning, practicing and publishing with TURK-MUSCULUS. J Back Musculoskelet Rehabil 2014;27:117-124.

Sutter EG, Widmyer MR, Utturkar GM, Spritzer CE, Garrett WE Jr, DeFrate LE. In vivo measurement of localized tibiofemoral cartilage strains in response to dynamic activity. Am J Sports Med 2015;43:370376.

Tuna S, Balcı N, Özçakar L. The relationship between femoral cartilage thickness and muscle strength in knee osteoarthritis. Clin Rheumatol 2016;35:2073-2077. 